\title{
Coexistence of Substance Abuse among Emergency Department Patients Presenting with Suicidal Ideation
}

\author{
Allison Tadros $\mathbb{D}^{\mathrm{D}},{ }^{1}$ Melinda Sharon, ${ }^{1}$ Michael Crum, ${ }^{1}$ Ryan Johnson, ${ }^{1}$ Kimberly Quedado, ${ }^{1}$ \\ and Wei Fang ${ }^{2}$ \\ ${ }^{1}$ West Virginia University Department of Emergency Medicine, USA \\ ${ }^{2}$ West Virginia Clinical \& Translational Science Institute, USA \\ Correspondence should be addressed to Allison Tadros; atadros@hsc.wvu.edu
}

Received 26 August 2019; Revised 3 March 2020; Accepted 23 September 2020; Published 29 September 2020

Academic Editor: Hideo Inaba

Copyright ( 2020 Allison Tadros et al. This is an open access article distributed under the Creative Commons Attribution License, which permits unrestricted use, distribution, and reproduction in any medium, provided the original work is properly cited.

\begin{abstract}
Background. Patients who are suicidal commonly seek care in the emergency department (ED). Few studies have examined the coexistence between suicidal ideation, substance abuse, and psychiatric diagnosis. Objectives. This study sought to determine how often suicidal ED patients have coexisting substance abuse and psychiatric diagnosis in addition to describing the characteristics of target population. Methods. In this retrospective cohort study, chart reviews were conducted of patients over 12 with suicidal ideation who presented to an academic ED from October 2016 to March 2017. Data abstracted included gender, age, insurance, prior psychiatric diagnoses, substances abused, presence of a suicide attempt, prior suicidality, number of ED visits in the prior year, and disposition. Both descriptive and inferential statistics were calculated. Results. There were 427 patient visits to the ED for suicidality during the study period, of which $54 \%$ were male, with a mean age of 34 years. Most patients (92\%) had a psychiatric diagnosis, most commonly depression (67\%). More than one psychiatric diagnosis was reported in $51 \%$ of patients, while $8 \%$ had no reported underlying psychiatric diagnosis. Substance abuse was reported in $58 \%$ of patients, including marijuana (42\%) and opioids (41\%). Polysubstance abuse was reported in $42 \%$. Approximately half of the patients had three or more ED visits in the previous 12 months. Most patients were insured by Medicaid (51\%), while 59\% were admitted for inpatient treatment. Conclusion. Substance abuse and psychiatric diagnosis were reported frequently among patients presenting to the ED with suicidal ideation, often involving more than one substance/diagnosis. Future studies should be aimed at evaluating the relationship between these conditions and determining how to better care for this population.
\end{abstract}

\section{Introduction}

Suicide deaths increased by more than 30\% from 1999 to 2017 and now represent the 10th leading cause of death in the United States (US) $[1,2]$. Suicide rates have increased in every age group under 75 years of age, with rural counties seeing much greater increases than urban areas [1]. Similarly, deaths attributable to drug overdose have dramatically increased during this century. From the same time period of 1999-2017, overdose deaths from prescription opioids increased five times [3]. Similarly, deaths from drug overdoses rates also have increased more dramatically in rural areas [4].

Recent studies have suggested that approximately $4 \%$ of adults in the US have suicidal thoughts annually $[5,6]$.
Patients with suicidal ideation or suicide attempt commonly present to the emergency department (ED) for evaluation, treatment, and possible placement into a psychiatric facility $[7,8]$. The number of patients presenting to the ED for suicidal thoughts has recently increased [8]. In 2013, patients with suicidal ideation represented $1 \%$ of all ED visits [8].

West Virginia has seen particularly high rates of substance abuse in the past decade and currently has the highest death rate from drug overdoses in the US at 58 per 100,000 residents [9-11]. West Virginia also has high suicide mortality rates, ranking 8 th highest in the country [12]. Despite rising rates of substance abuse, overdose deaths, and suicidal ideation, few studies have examined the coexistence between substance abuse, psychiatric diagnoses, and suicidality. The purpose of this study was to determine how often patients 
seeking assistance in a West Virginia ED for suicidal ideation or suicide attempt have coexisting substance abuse. In addition, the specific substances being abused were determined. Comparisons were made with substance abuse and psychiatric diagnoses between males and females.

\section{Materials and Methods}

2.1. Study Design, Setting, and Patient Selection. This study was conducted at a tertiary care academic ED with an annual census of approximately 50,000 visits. Both pediatric and adult patients are cared for at this facility. We performed a retrospective cohort study of patients aged 13 years and older who visited our ED between October 1, 2016, and March 31, 2017, who were diagnosed by ICD-10 codes with either R45.851 (suicidal ideation) or T14.91 (suicide attempt).

2.2. Data Collection and Analyses. The charts of patients meeting the inclusion criteria were reviewed by 3 of the authors (AT, RJ, and MC). Data abstracted included age, gender, number of ED visits in the prior 12 months, current substances being used at the time of visit, insurance status, disposition, whether the patient self-reported any prior suicide attempt(s), previously diagnosed psychiatric disorders, and if they reported a suicide attempt immediately preceding the current visit. One reviewer (AT) reviewed approximately $50 \%$ of the other reviewers' charts for consistency purposes.

Descriptive statistics regarding age, gender, substances, psychiatric diagnoses, number of ED visits, and disposition were calculated. Regarding the frequency of substance use or psychiatric diagnosis, if a patient was indicated to use more than one substance, they were listed in a polysubstance category; otherwise, their case was limited to the single substance or no substance; synonymously, if a patient had more than one psychiatric diagnosis, they were separated into a multiple diagnosis category. If there was one or no diagnosis, they remained in their specific diagnosis category as well. In addition, Fisher's exact test was conducted to determine whether there is a significant difference in the distribution of either single substance abuse or polysubstance abuse cases between males and females and whether there is a significant difference in the distribution of either single psychiatric diagnosis or multiple psychiatric diagnoses between males and females. All statistical analysis was conducted using SAS 9.4 (The SAS Institute, Cary, NC). This study was approved by the university's Institutional Review Board.

\section{Results}

From October 1, 2016, to March 31, 2017, there were 427 patients seen in the ED with suicidal ideation or suicide attempt. Males represented 53\% of the total sample, with an average age of 34 years, ranging from 13 to 84 years. Medicaid was the primary insurer for $51 \%$ of the sample.

Overall, $51 \%$ of the patients were admitted to a psychiatric facility or the hospital (Table 1 ).

In addition to suicidal ideation or attempt, $59 \%$ of the patients also reported abuse of at least one substance. Substance abuse was reported in $56 \%$ of males and $46 \%$ of
TABLE 1: Demographic characteristics of patients presenting to the emergency department (ED) for suicidality.

\begin{tabular}{lc}
\hline Characteristic & $N(\%)$ \\
\hline Total & 427 \\
Gender & \\
Male & $228(53)$ \\
Female & $199(47)$ \\
Age (years) & \\
Average & 34 \\
Range & $13-84$ \\
Payor type & \\
Medicare & $59(14)$ \\
Medicaid & $216(51)$ \\
Private & $142(33)$ \\
Self-pay & $6(1.1)$ \\
Uninsured & $3(0.7)$ \\
Other & $1(0.2)$ \\
Coexisting substance abuse & \\
Yes & $250(59)$ \\
No & $177(41)$ \\
Underlying psychiatric diagnosis & \\
Yes & $392(92)$ \\
No & $35(8)$ \\
ED disposition & \\
Admit & $253(59)$ \\
Discharge & $173(40)$ \\
Eloped & $1(1)$ \\
\hline
\end{tabular}

females. The most commonly abused substances in males were opioids and marijuana, while $28 \%$ reported polysubstance abuse (Table 2). Females most commonly abused opioids and marijuana as well, with $21 \%$ reporting the abuse of more than one substance (Table 2). Of patients with suicidal ideation or attempt who had either no substance abuse or abused only one substance, there was no statistical difference in the distribution of substance which was being used between men and women ( $p$ value $=0.1595)$. Likewise, there were no gender differences found in the distribution of substances being abused in patients with polysubstance abuse $(p$ value $=0.5470)$

Underlying psychiatric disorder(s) were common among patients with suicidal ideation or attempt but was not present in all patients (Tables 1 and 3). Depression and anxiety were the most commonly reported psychiatric diagnoses in both men and women, with $47 \%$ of men and $49 \%$ of women suffered from more than one disorder (Table 3 ). Only $8 \%$ of the patients with suicidal ideation did not have an underlying psychiatric diagnosis, while $5 \%$ of the patients reported no substance abuse or underlying psychiatric diagnosis. Further information on underlying psychiatric disorders among the patients presenting with suicidal ideation or attempt can be seen in Table 3. Of the patients with no psychiatric diagnosis or only one psychiatric diagnosis, there was no statistically 
TABLE 2: Gender comparisons of self-reported substances. Substances may be reported both individually and under polysubstance if applicable.

\begin{tabular}{lcc}
\hline & Males (\%) & Females (\%) \\
& $N=228$ & $N=199$ \\
\hline Alcohol & $35(15)$ & $11(6)$ \\
Amphetamines & $29(13)$ & $23(12)$ \\
Barbiturates & $1(0.4)$ & $0(0)$ \\
Bath salts & $0(0)$ & $1(0.5)$ \\
Benzo & $30(13)$ & $24(12)$ \\
Buprenorphine & $0(0)$ & $1(0.5)$ \\
Cocaine & $3(1.3)$ & $1(0.5)$ \\
LSD & $1(0.4)$ & $1(0.5)$ \\
Marijuana & $63(28)$ & $43(22)$ \\
Opioid & $59(26)$ & $47(24)$ \\
Polysubstances & $63(28)$ & $42(21)$ \\
None & $100(44)$ & $107(54)$ \\
\hline
\end{tabular}

TABLE 3: Gender comparisons of psychiatric diagnoses. Diagnoses may be reported both individually and under multiple diagnoses. (Note: males $n=228$, females $n=199$ ).

\begin{tabular}{lcc}
\hline & Male (\%) & Female (\%) \\
\hline ADHD & $25(11)$ & $16(8.0)$ \\
Anxiety & $69(30)$ & $92(46)$ \\
Bipolar & $27(12)$ & $15(7.5)$ \\
Depression & $136(60)$ & $132(66)$ \\
OCD & $0(0)$ & $1(0.5)$ \\
ODD & $2(0.9)$ & $0(0)$ \\
PTSD & $37(16)$ & $41(21)$ \\
Thought disorder & $36(16)$ & $9(4.5)$ \\
Multiple diagnoses & $108(47)$ & $97(49)$ \\
None & $18(7.9)$ & $16(8)$ \\
\hline
\end{tabular}

significant difference in the distribution or diagnosis between men and women $(p$ value $=0.1170)$.

Several patients included in the study had made more than one visit in the prior 12 months to the same ED. Frequent visits were particularly common in the older age groups (Table 4). Although patients over 50 years of age comprised only $19 \%$ of the total population of visits for suicidal ideation, $24 \%$ of them had over 10 visits in a year.

\section{Discussion}

Patients with substance abuse and suicidal ideation are both commonly encountered in EDs [13-15]. Mental health and substance abuse disorders account for approximately one out of every eight ED visits in the US and are commonly encountered in the same patient populations $[15,16]$. Suicide represents a growing public health concern and is currently the $10^{\text {th }}$ leading cause of death in WV [11]. Suicide is also the leading cause of death among people with substance use disorders [17]. The relationship between substance abuse and increased risk of suicide is well demonstrated in both the psychiatric and public health literatures. Given the prevalence of these diagnoses in ED patients, this study attempted to quantify the frequency of substance abuse in patients with suicidal ideation or attempt.

This study found that substance abuse was relatively common in ED patients presenting with suicidal ideation, with over half of the patients with suicidal ideation in the ED also reporting substance abuse. The most commonly abused substances among both men and women were marijuana and opioids, while over $20 \%$ of patients abused more than one substance. Of note, this study was conducted in a state where marijuana use is currently illegal. The percentage of patients self-reporting only alcohol use was relatively low at $17 \%$, which is possibly the result of patients using alcohol in addition to other substances [18]. Although prior studies have found gender differences in substance abuse, statistically significant differences were not found within our patient cohort $[19,20]$.

In 2015, there were an estimated 5.7 million ED visits in the US, with the primary diagnosis of a psychiatric disorder [21]. In the current study, it is not surprising that the vast majority had at least one existing psychiatric diagnosis. Mood disorders were most commonly seen, with $67 \%$ reporting depression and $40 \%$ suffering from anxiety. Roughly half of the study population had multiple psychiatric diagnoses, and over one-third of patients had a history of at least one prior suicide attempt. Other studies have also found frequent recurrence of suicidal ideation and attempts, with a higher likelihood in patients with concomitant substance abuse $[22,23]$. This data highlights the need for ongoing care and the potentially recurrent nature of psychiatric illness in patients with suicidal thoughts and substance abuse.

Almost half of the patients presenting for suicidal ideation had three or more visits to the same ED within the previous 12 months, and there were many patients who had over 10 visits in the prior year. These encounters only include visits to our facility and do not account for possible visits to other facilities. A previous study found that $14 \%$ of the patients with an ED visit for mental health or substance abuse had a return visit to the ED or hospitalization within 30 days, particularly for older adults [22]. Other studies have similarly reported that patients with both a psychiatric and substance abuse diagnoses had significantly higher ED utilization [14, 24-26]. These findings highlight the chronic nature of substance abuse and psychiatric illness and therefore the need for adequately resourced psychiatric care programs.

Males were more commonly seen in our ED for suicidal ideation than females. This is in contrast to an earlier study which found a slight female prevalence [27]. Medicaid was the payer for over half of the visits in this study, followed by private insurance. A study examining ED visits nationally for suicidal ideation reported that Medicaid was the insurer for $28 \%$ of patients, with over $25 \%$ of these patients being uninsured [27]. The present study had nearly double the amount of patients on Medicaid as compared to this brief report. However, that study was conducted from 2006-2013 and therefore included data prior to the Affordable Care Act. 
TABLE 4: Number of ED visits in the past six months by patients grouped by age (in years).

\begin{tabular}{|c|c|c|c|c|c|c|}
\hline & 1 to $2(\%)$ & 3 to $4(\%)$ & 5 to $6(\%)$ & 7 to $10(\%)$ & More than $10(\%)$ & Total (\%) \\
\hline Less than 18 & $51(77)$ & $10(15)$ & $4(6.5)$ & $1(1.5)$ & $0(0)$ & $66(15)$ \\
\hline 18 to 29 & $80(57)$ & $22(16)$ & $16(11)$ & $9(6.1)$ & $14(9.9)$ & $141(33)$ \\
\hline 30 to 49 & $63(45)$ & $20(14)$ & $10(7.1)$ & $11(7.9)$ & $36(26)$ & $140(33)$ \\
\hline 50 and older & $37(47)$ & $16(20)$ & $5(6.5)$ & $2(2.5)$ & $19(24)$ & 79 (19) \\
\hline
\end{tabular}

Although the majority of patients in the present study were admitted to an inpatient treatment setting, a substantial minority of patients (40\%) were discharged from the ED with a plan to establish or continue existing outpatient treatment. In comparison, a national study reported that $27 \%$ of this patient population was discharged upon their ED visit [27]. It is worth further examining in future research what factors contribute to this patient population's disposition and how suicidal ideation and/or substance abuse affects this decision.

4.1. Limitations. There are several limitations to this study that warrant further discussion. First, this study was conducted at a single ED, limiting the diversity of the studied patient population, and potentially limits the generalizability. Factors such as the disproportionately higher rates of opioid abuse among this state population when compared to the national rate may contribute to this [10]. Second, this study was a retrospective chart review and was subjected to the inherent limitations of this type of study, including potential selection, recall, and misclassification biases. Another potential limitation was variability in data collection due to factors including inconsistencies of the electronic medical record, variability in provider documentation, and variation between data abstracter methods. Lastly, another important limitation was the reliance on either patient self-reporting or the use of potentially inaccurate urine drug screen results to classify patient substance abuse status.

\section{Conclusions}

This study further illustrated that substance abuse is commonly encountered in emergency department patients with suicidal ideation. Polysubstance abuse and suffering from multiple psychiatric diagnoses were also relatively common. Caring for these patients is complex and multifaceted. Further research is needed to analyze the relationship between suicidality, gender, substance abuse, and mental health as well as how to best serve this population. In particular, studies to determine if the treatment of substance abuse leads to a reduction in suicidal ideation and attempt would be useful to practitioners.

\section{Data Availability}

The collection of the data for this research paper required the authors to enter patient medical records, with approval of our Institutional Review Board. This data would therefore only be available to the authors.

\section{Disclosure}

The content is solely the responsibility of the authors and does not necessarily represent the official views of the National Institutes of Health.

\section{Conflicts of Interest}

The authors, Tadros, Sharon, Crum, Johnson, Quedado, and Fang, declare that there is no conflict of interest to report.

\section{Acknowledgments}

Dr. Wei Fang, who provided statistical support for this publication, is funded through the WVCTSI. The research reported in this publication was supported by the National Institute of General Medical Sciences of the National Institutes of Health under Award Number 5U54GM104942-04.

\section{References}

[1] Centers for Disease Control and Prevention, Suicide mortality in the United States, 19992017. NCHS Data Brief No. 330, November 2018April 2019, https://www.cdc.gov/nchs/ products/databriefs/db330.htm.

[2] S. C. Curtin, M. Warner, and H. Hedegaard, Increase in suicide in the United States, 1999-2014. NCH Data Brief. 2016 Apr;241:1-8. American Foundation for Suicide Prevention. Suicide statistics. $2017 \mathrm{https}$ //afsp.org/about-suicide/ suicidestatistics/.

[3] Centers for Disease Control and Prevention, Prescription opioid data. 2018April 2019, https://www.cdc.gov/drugoverdose/ data/prescribing.html.

[4] Centers for Disease Control and Prevention, CDC reports rising rates of drug overdose deaths in rural areas. 2017April 2019, https://www.cdc.gov/media/releases/2017/p1019-ruraloverdose-deaths.html.

[5] US Department of Health and Human Services, Results from the 2013 national survey on drug use and health: mental health findings. 2014April 2019, https://www.samhsa.gov/data/sites/ default/files/NSDUHmhfr2013/NSDUHmhfr2013.pdf.

[6] K. Piscopo, R. N. Lipari, J. Cooney, and C. Glasheen, "Suicidal thoughts and behavior among adults: results from the 2015 National Survey on Drug Use and Health," NSDUH Data Review, 2016, https://www.samhsa.gov/data/sites/default/ files/NSDUH-DRFFR32015/NSDUH-DR-FFR3-2015.htm.

[7] A. Vandyk, A. Bentz, S. Bissonette, and C. Cater, "Why go to the emergency department? Perspectives from persons with borderline personality disorder," International Journal of Mental Health Nursing, vol. 28, no. 3, pp. 757-765, 2019, [Epub ahead of print]. 
[8] P. L. Owens, K. R. Fingar, K. C. Heslin, R. Mutter, and C. L. Booth, Emergency department visits related to suicidal ideation, 2006-2013: statistical brief \#220, 2017, April 2019, https://www.ncbi.nlm.nih.gov/books/NBK442036/.

[9] National Institute on Drug Abuse, Opioid summaries by state, 2019, April 2019, https://www.drugabuse.gov/drug-topics/ opioids/opioid-summaries-by-state.

[10] Centers for Disease Control and Prevention, Drug overdose deaths, 2018, April 2019, https://www.cdc.gov/drugoverdose/ data/statedeaths.html.

[11] Centers for Disease Control and Prevention, Stats of the State of West Virginia, 2018, May 2019, https://www.cdc.gov/nchs/ pressroom/states/westvirginia/westvirginia.htm.

[12] B. Chakravarthy, S. Toohey, Y. Rezaimehr et al., "National differences between ED and ambulatory visits for suicidal ideation and attempts and depression," The American Journal of Emergency Medicine, vol. 32, no. 5, pp. 443-447, 2014, Epub 2013 Dec 26.

[13] M. W. Smith, C. Stocks, and P. B. Santora, "Hospital readmission rates and emergency department visits for mental health and substance abuse conditions," Community Mental Health Journal, vol. 51, no. 2, pp. 190-197, 2015, Epub 2015 Jan 7.

[14] A. J. Weiss, A. L. Barrett, K. C. Heslin, and C. Stocks, Trends in emergency department visits involving mental and substance use disorders, 2006-2013: statistical brief \#216, 2016, April 2019, https://www.hcup-us.ahrq.gov/reports/statbriefs/sb216Mental-Substance-Use-Disorder-ED-Visit-Trends.jsp.

[15] H. M. Pettinati, C. P. O’Brien, and W. D. Dundon, "Current status of co-occurring mood and substance use disorders: a new therapeutic target," The American Journal of Psychiatry, vol. 170, no. 1, pp. 23-30, 2013.

[16] Center for Substance Abuse Treatment, Substance abuse and suicide prevention: evidence and implications - a white paper. DHHS Pub. No. SMA-08-4352, Substance Abuse and Mental Health Services Administration, Rockville, MD, 2008.

[17] F. S. Stinson, B. F. Grant, D. A. Dawson, W. J. Ruan, B. Huang, and T. Saha, "Comorbidity between DSM-IV alcohol and specific drug use disorders in the United States: results from the National Epidemiologic Survey on Alcohol and Related Conditions," Drug and Alcohol Dependence, vol. 80, no. 1, pp. 105-116, 2005, Epub 2005 Apr 18.

[18] February 2020, https://www.samhsa.gov/data/sites/default/ files / Revised $2 \mathrm{k} 11 \mathrm{NS}$ DUHS u m N at Finding / Revised2k11NSDUHSummNatFindings/NSDUHresults2011 .htm\#2.5.

[19] https://wwwdasis.samhsa.gov/dasis2/teds_pubs/TEDS-2017R.pdf.

[20] Centers for Disease Control and Prevention, Mental health. 2017April 2019, https://www.cdc.gov/nchs/fastats/mentalhealth.htm.

[21] Y. Liu, J. Sareen, J. M. Bolton, and J. L. Wang, "Development and validation of a risk prediction algorithm for the recurrence of suicidal ideation among general population with low mood," Journal of Affective Disorders, vol. 193, pp. 11-17, 2016, Epub 2015 Dec 31.

[22] E. N. Dugas, N. C. Low, E. K. O’Loughlin, and J. L. O’Loughlin, "Recurrent suicidal ideation in young adults," Canadian Journal of Public Health, vol. 106, no. 5, pp. e303-e307, 2015.

[23] S. Lee, J. Herrin, W. V. Bobo, R. Johnson, L. Sangaralingham, and R. Campbell, "Predictors of return visits among insured emergency department mental health and substance abuse patients, 2005-2013," The Western Journal of Emergency Medicine, vol. 18, no. 5, pp. 884-893, 2017, Epub 2017 Jul 17.

[24] G. M. Curran, G. Sullivan, K. Williams et al., "Emergency department use of persons with comorbid psychiatric and substance abuse disorders," Annals of Emergency Medicine, vol. 41, no. 5, pp. 659-667, 2003.

[25] Y. Ledoux and P. Minner, "Occasional and frequent repeaters in a psychiatric emergency room," Social Psychiatry and Psychiatric Epidemiology, vol. 41, no. 2, pp. 115-121, 2006, Epub 2006 Jan 31.

[26] M. Schmidt, "Frequent visitors at the psychiatric emergency room - a literature review," The Psychiatric Quarterly, vol. 89, no. 1, pp. 11-32, 2018.

[27] B. Chakravarthy, W. Hoonpongsimanont, C. L. Anderson, M. Habicht, T. Bruckner, and S. Lotfipour, "Depression, suicidal ideation, and suicidal attempt presenting to the emergency department: differences between these cohorts," The Western Journal of Emergency Medicine, vol. 15, no. 2, pp. 211-216, 2014. 